CERN-PH-TH/2007-162

SLAC-PUB-12817

KEK-TH-1173

LAL 07-175

October 2007

\title{
On the Physics Case of a Super Flavour Factory
}

\author{
T.Browder, ${ }^{1}$ M.Ciuchini, ${ }^{2}$ T.Gershon, ${ }^{3}$ M.Hazumi, ${ }^{4,7}$ T.Hurth,${ }^{5,6}$ Y.Okada, ${ }^{4,7}$ A.Stocchi ${ }^{8,9}$ \\ ${ }^{1}$ University of Hawaii at Manoa, Honolulu, Hawaii 96822, USA \\ ${ }^{2}$ INFN Sezione di Roma Tre and Dip. di Fisica, Univ. of Roma Tre, I-00146 Rome, Italy \\ ${ }^{3}$ University of Warwick, Department of Physics, Coventry CV4 7AL, UK \\ ${ }^{4}$ High Energy Accelerator Research Organization (KEK), Tsukuba, Japan \\ ${ }^{5}$ CERN, Department of Physics, Theory Unit, CH-1211 Geneva 23, Switzerland \\ ${ }^{6}$ SLAC, Stanford University, Stanford, CA 94309, USA \\ ${ }^{7}$ Graduate University for Advanced Studies (Sokendai), Tsukuba, Japan \\ ${ }^{8}$ Laboratoire de l'Accelerateur Lineaire IN2P3-CNRS, France \\ ${ }^{9}$ University de Paris-Sud, BP34, F-91898 Orsay cedex, France
}

\begin{abstract}
We summarize the physics case of a high-luminosity $e^{+} e^{-}$flavour factory collecting an integrated luminosity of $50-75 \mathrm{ab}^{-1}$. Many New Physics sensitive measurements involving $B$ and $D$ mesons and $\tau$ leptons, unique to a Super Flavour Factory, can be performed with excellent sensitivity to new particles with masses up to $\sim 100$ (or even $\sim 1000$ ) TeV. Flavour- and $C P$-violating couplings of new particles that may be discovered at the LHC can be measured in most scenarios, even in unfavourable cases assuming minimal flavour violation. Together with the LHC, a Super Flavour Factory, following either the SuperKEKB or the Super $B$ proposal, could be soon starting the project of reconstructing the New Physics Lagrangian.
\end{abstract}




\section{Introduction}

Many open fundamental questions of particle physics are related to flavour: How many families are there? What is their origin? How are neutrino and quark masses and mixing angles generated? Do there exist new sources of flavour and $C P$ violation beyond those we already know? What is the relation between the flavour structure in the lepton and quark sectors? Future flavour experiments will attempt to address these questions providing the exciting possibility to learn something about physics at energy scales much higher than those reachable by current experiments.

The Standard Model (SM) of elementary particles has been very successful in explaining a wide variety of existing experimental data. It accounts for a range of phenomena from low-energy physics (less than a $\mathrm{GeV}$ ), such as kaon decays, to high-energy (a few hundred $\mathrm{GeV}$ ) processes involving real weak gauge bosons ( $W$ and $Z$ ) and top quarks. There is, therefore, little doubt that the SM is the theory to describe physics below the energy scale of several hundred $\mathrm{GeV}$, namely all that has been explored so far.

In spite of the tremendous success of the SM, it is fair to say that the flavour sector of the SM is much less understood than its gauge sector, reflecting our lack of answers to the questions mentioned above. Masses and mixing of the quarks and leptons, which have a significant but unexplained hierarchy pattern, enter as free parameters to be determined experimentally. In fact, while symmetries shape the gauge sector, no principle governs the flavour structure of the SM Lagrangian. Yukawa interactions provide a phenomenological description of the flavour processes which, while successful so far, leaves most fundamental questions unanswered. Hence the need to go beyond the SM.

Indeed the search for evidence of physics beyond the SM is the main goal of particle physics in the next decades. The LHC at CERN will start soon looking for the Higgs boson, the last missing building block of the SM. At the same time it will intensively search for New Physics (NP), for which there are solid theoretical motivations related to the quantum stabilization of the Fermi scale to expect an appearance at energies around $1 \mathrm{TeV}$.

However, pushing the high-energy frontier, i.e. increasing the available centre-of-mass energy in order to produce and observe new particles, is not the only way to look for NP. New particles could reveal themselves through their virtual effects in processes involving only standard particles as has been the case several times in the history of particle physics. For these kind of searches the production thresholds are not an issue. Since quantum effects become typically smaller as the mass of the virtual particles increases, the name of the game is rather high precision. As a matter of fact, high-precision measurements probe NP energy scales inaccessible at present and next-generation colliders at the energy frontier.

Flavour physics is the best candidate as a tool for NP searches through quantum effects for several reasons. Flavour Changing Neutral Currents (FCNC), neutral meson-antimeson mixing and $C P$ violation occur at the loop level in the SM and therefore are potentially subject to $\mathcal{O}(1)$ NP virtual corrections. In addition, quark flavour violation in the SM is governed by the weak interaction and suppressed by the small quark mixing angles. Both these features are not necessarily shared by NP which, in such cases, could produce very large effects. Indeed, the inclusion in the SM of generic NP flavour-violating terms with natural $\mathcal{O}(1)$ couplings is known to violate present experimental constraints unless the NP scale is pushed up to 10-100 TeV depending on the flavour sector. This difference between the NP scale emerging from flavour physics and the one suggested by Higgs physics could be a problem for model builders (the so-called flavour problem), but it clearly indicates that flavour physics has the potential to push the explored NP scale in the $100 \mathrm{TeV}$ region. On the other hand, if the NP scale is indeed close to $1 \mathrm{TeV}$, the flavour structure of NP must be highly non-trivial and the experimental determination of the flavour-violating couplings is particularly interesting.

Let us elaborate on this latter option. Any new-physics model, established at the TeV scale to solve the gauge hierarchy problem, includes new flavoured particles and new flavour- and $C P$-violating parameters. Therefore, such a model must provide a solution also to the flavour and $C P$ problems, 
namely how new flavour changing neutral currents and $C P$-violating phenomena are suppressed. This may be related to other interesting questions. For instance, in supersymmetry the flavour problem is directly linked to the crucial issue of supersymmetry breaking. Similar problems also occur in models of extra-dimensions (flavour properties of Kaluza-Klein states), Technicolour models (flavour couplings of Techni-fermions), little-Higgs models (flavour couplings of new gauge bosons and fermions) and multi-Higgs models ( $C P$-violating Higgs couplings). Once NP is found at the TeV scale, precision measurements of flavour- and $C P$-violating observables would shed light on the detailed structure of the underlying model.

On quite general grounds, quantum effects in flavour processes explore a parameter space including the NP scale and the NP flavour- and $C P$-violating couplings. In specific models these are related to fundamental parameters such as masses and couplings of new particles. In particular, NP effects tend to disappear at large NP scales as well as for small couplings. Therefore a crucial question is: could NP be flavour-blind, thus making searches for it with flavour physics unfeasible? Fortunately, the concept of Minimal Flavour Violation (MFV) provides a negative answer: even if NP does not contain new sources of flavour and $C P$ violation, the flavour-violating couplings present in the SM are enough to produce a new phenomenology that makes flavour processes sensitive to the presence of new particles. In other words, MFV puts a lower bound on the flavour effects generated by NP appearing at a given mass scale, a sort of "worst case" scenario for the flavour-violating couplings extremely useful to exclude NP flavourblindness and assess the "minimum" performance of flavour physics in searching for NP, always keeping in mind that larger effects are quite possible and easily produced in many scenarios beyond MFV.

In the light of the above considerations, a Super Flavour Factory (SFF), following the recent SuperKEKB [1] and Super $B$ [2] proposals, has one mission: to search for new physics in the flavour sector exploiting a huge leap in integrated luminosity and the wide range of observables that it can measure. However this goal can be pursued in different ways depending on whether evidence of NP has been found at the time a SFF starts taking data.

In either scenario, a SFF can search for evidence of NP irrespective of the values of the new particle masses and of the unknown flavour-violating couplings. A large number of measurements could provide evidence for NP at a SFF. A first set is given by measurements of observables which are predicted by the SM with small uncertainty, including those which are vanishingly small (the so-called null tests). Among them are the flavour-violating $\tau$ decays, direct $C P$ asymmetries in $B \rightarrow X_{s+d} \gamma$, in $\tau$ decays and in some non-leptonic $D$ decays, $C P$ violation in neutral charm meson mixing, the dilepton invariant mass at which the forward-backward asymmetry of $B \rightarrow X_{s} \ell^{+} \ell^{-}$vanishes, and lepton universality violating $B$ and $\tau$ decays. Any deviation, as small as a SFF could measure, from its SM value of any observable in this set could be ascribed to NP with essentially no uncertainty. A second set of NPsensitive observables, including very interesting decays such as $b \rightarrow s$ penguin-dominated non-leptonic $B$ decays, $B \rightarrow \tau \nu, B \rightarrow D^{(*)} \tau \nu, B \rightarrow K^{*} \gamma, B \rightarrow \rho \gamma$, and many others, require more accurate determinations of SM contributions and improved control of the hadronic uncertainties with respect to what we can do today in order to match the experimental precision achievable at a SFF and to allow for an unambiguous identification of a NP signal. The error on the SM can be reduced using the improved determination of the Cabibbo-Kobayashi-Maskawa (CKM) matrix provided by a SFF itself. This can be achieved using generalized CKM fits which allow for a 1\% determination of the CKM parameters using tree-level and $\Delta F=2$ processes even in the presence of generic NP contributions. As far as hadronic uncertainties are concerned, the extrapolation of our present knowledge and techniques shows that it is possible to reach the required accuracy by the time a SFF will be running using improved lattice QCD results obtained with next-generation computers [2] and/or bounding the theoretical uncertainties with data-driven methods exploiting the huge SFF data sample.

As we already noted, the NP search at a SFF could reveal the virtual effect of particles with masses of hundreds of $\mathrm{TeV}$ and in some cases, notably $\Delta F=2$ processes, even thousands of $\mathrm{TeV}$ depending on the values of the flavour-violating couplings. Therefore this search is worth doing irrespective of 
whether NP has already been found or not. If new particles are discovered at the energy frontier, a SFF could enlarge the spectrum providing evidence of heavier states not accessible otherwise; if not, quantum effects measurable at a SFF could be the only option to look for NP for a long time.

If the LHC finds NP at the TeV scale - in particular if the findings include one (or more) new flavoured particle(s) - then a SFF could measure its flavour- and $C P$-violating couplings. Indeed all terms of the NP Lagrangian non-diagonal in the flavour space are barely accessible at the LHC. A SFF would be needed to accomplish the task of reconstructing them. It seems able to do that even in the unfavourable cases provided by most MFV models. Indeed, for the purpose of inferring the NP Lagrangian from experiments, the LHC and SFF physics programmes are complementary.

Finally, it must be emphasised that while a Super Flavour Factory will perform detailed studies of beauty, charm and tau lepton physics, the results will be highly complementary to those on several important observables related to $B_{s}$ meson oscillations, kaon and muon decays that will be measured elsewhere. Most benchmark charm measurements, in particular interesting NP-related measurements such as $C P$ violation in charm mixing, will still be statistics-limited after the CLEO $c$, BESIII and $B$ factory projects are completed, and can only be pursued to their ultimate precision at a SFF. Operation at the $\Upsilon(5 \mathrm{~S})$ resonance provides the possibility of exploiting the clean $e^{+} e^{-}$environment to measure $B_{s}^{0}$ decays with neutral particles in the final state, which will complement the channels that can be measured at LHCb. A SFF has sensitivity for $\tau$ physics that is far superior to any other existing or proposed experiment, and the physics reach can be extended even further by the possibility to operate with polarized beams. It is particularly noteworthy that the combined information on $\mu$ and $\tau$ flavour violating decays that will be provided by MEG [3] together with a SFF can shed light on the mechanism responsible for lepton flavour violation.

\section{Experimental Sensitivities}

A Super Flavour Factory (SFF) with integrated luminosity of $50-75 \mathrm{ab}^{-1}$ can perform a wide range of important measurements and dramatically improve upon the results from the current generation of $B$ Factories. Many of these measurements cannot be made in a hadronic environment, and are unique to a SFF. The experimental sensitivities of a SFF can be schematically classified in two categories:

- Searching for New Physics:

Many of the measurements that can be made at a SFF are highly sensitive to NP effects, and those with precise SM predictions are potential discovery channels. As an example: the mixinginduced $C P$ asymmetry parameter for $B^{0} \rightarrow \phi K^{0}$ decays can be measured to a precision of 0.02 , as can equivalent parameters for numerous hadronic decay channels dominated by the $b \rightarrow s$ penguin transition. These constitute very stringent tests of any NP scenario which introduces new $C P$ violation sources, beyond the Standard Model. The presence of new sources of $C P$ violation in $D^{0}-\bar{D}^{0}$ mixing, where the SM background is negligible, can be tested to similar precision. New physics that appears in the $D^{0}$ sector (involving up-type quarks) may be different or complementary to that in the $B_{d}^{0}$ or $B_{s}^{0}$ sectors. Direct $C P$ asymmetries can be measured to the fraction of a percent level in $b \rightarrow s \gamma$ decays, using both inclusive and exclusive channels, and $b \rightarrow s \ell^{+} \ell^{-}$can be equally thoroughly explored. Equally precise searches for direct $C P$ violation in charm or $\tau$ decays provide additional NP sensitivity, since the SM background is largely absent. At the same time, a SFF can access channels that are sensitive to NP even when there are no new sources of $C P$ violation, such as the photon polarization in $b \rightarrow s \gamma$, and the branching fractions of $B^{+} \rightarrow \ell^{+} \nu_{\ell}$, the latter being sensitive probes of NP in MFV scenarios with large $\tan \beta$. Furthermore, rare FCNC decays of the $\tau$ lepton are particularly interesting since lepton flavour violation sources involving the third generation are naturally the largest. Any of these measurements constitutes clear motivation for a SFF. 
- Future metrology of the CKM matrix:

There are several measurements that are unaffected by NP in many likely scenarios, and which allow the extraction of the CKM parameters even in the presence of such NP effects. Among these, the angle $\gamma$ can be measured with a precision of $1-2^{\circ}$, where the precision is limited only by statistics, not by systematics or by theoretical errors. By contrast, the determination of the elements $\left|V_{u b}\right|$ and $\left|V_{c b}\right|$ will be limited by theory, but the large data sample of a SFF will allow many of the theoretical errors to be much improved. With anticipated improvements in lattice QCD calculations, the precision on $\left|V_{u b}\right|$ and $\left|V_{c b}\right|$ can be driven down to the percent level. These measurements could allow tests of the consistency of the Standard Model at a few per mille level and provide the NP phenomenological analyses with a determination of the CKM matrix at the percent level.

In Table 1 we give indicative estimates of the precision on some of the most important observables that can be achieved by a SFF with integrated luminosity of 50-75 $\mathrm{ab}^{-1}$. Here we have not attempted to comment on the whole range of measurements that can be performed by such a machine, but instead focus on channels with the greatest phenomenological impact. For more details, including a wide range of additional measurements, we guide the reader to the reports $[1,2,4-6]$, where also all original references are given.

Table 1: Expected sensitivity that can be achieved on some of the most important observables, by a SFF with integrated luminosity of $50-75 \mathrm{ab}^{-1}$. The range of values given allow for possible variation in the total integrated luminosity, in the accelerator and detector design, and in limiting systematic effects. For further details, refer to $[2,6]$.

\begin{tabular}{lc}
\hline \hline Observable & Super Flavour Factory sensitivity \\
\hline $\sin (2 \beta)\left(J / \psi K^{0}\right)$ & $0.005-0.012$ \\
$\gamma\left(B \rightarrow D^{(*)} K^{(*)}\right)$ & $1-2^{\circ}$ \\
$\alpha(B \rightarrow \pi \pi, \rho \rho, \rho \pi)$ & $1-2^{\circ}$ \\
$\left|V_{u b}\right|($ exclusive $)$ & $3-5 \%$ \\
$\left|V_{u b}\right|$ (inclusive) & $2-6 \%$ \\
\hline $\bar{\rho}$ & $1.7-3.4 \%$ \\
$\bar{\eta}$ & $0.7-1.7 \%$ \\
\hline$S\left(\phi K^{0}\right)$ & $0.02-0.03$ \\
$S\left(\eta^{\prime} K^{0}\right)$ & $0.01-0.02$ \\
$S\left(K_{S}^{0} K_{S}^{0} K_{S}^{0}\right)$ & $0.02-0.04$ \\
\hline$\phi_{D}$ & $1-3^{\circ}$ \\
\hline $\mathcal{B}(B \rightarrow \tau \nu)$ & $3-4 \%$ \\
$\mathcal{B}(B \rightarrow \mu \nu)$ & $5-6 \%$ \\
$\mathcal{B}(B \rightarrow D \tau \nu)$ & $2-2.5 \%$ \\
\hline $\mathcal{B}(B \rightarrow \rho \gamma) / \mathcal{B}\left(B \rightarrow K^{*} \gamma\right)$ & $3-4 \%$ \\
$A_{C P}(b \rightarrow s \gamma)$ & $0.004-0.005$ \\
$A_{C P}(b \rightarrow(s+d) \gamma)$ & 0.01 \\
$S\left(K_{S}^{0} \pi^{0} \gamma\right)$ & $0.02-0.03$ \\
$S\left(\rho^{0} \gamma\right)$ & $0.08-0.12$ \\
$A^{\mathrm{FB}}\left(B \rightarrow X_{s} \ell^{+} \ell^{-}\right) s_{0}$ & $4-6 \%$ \\
$\mathcal{B}(B \rightarrow K \nu \bar{\nu})$ & $16-20 \%$ \\
\hline $\mathcal{B}(\tau \rightarrow \mu \gamma)$ & $2-8 \times 10^{-9}$ \\
$\mathcal{B}(\tau \rightarrow \mu \mu \mu)$ & $0.2-1 \times 10^{-9}$ \\
$\mathcal{B}(\tau \rightarrow \mu \eta)$ & $0.4-4 \times 10^{-9}$ \\
\hline \hline
\end{tabular}


The most important measurements within the CKM metrology are the angles of the Unitarity Triangle, the angle $\beta$ (also known as $\phi_{1}$ ), measured using mixing-induced $C P$ violation in $B^{0} \rightarrow J / \psi K^{0}$, the angle $\alpha\left(\phi_{2}\right)$, measured using rates and asymmetries in $B \rightarrow \pi \pi^{1}, \rho \pi$ and $\rho \rho$, and the angle $\gamma\left(\phi_{3}\right)$, measured using rates and asymmetries in $B \rightarrow D^{(*)} K^{(*)}$ decays, using final states accessible to both $D^{0}$ and $\bar{D}^{0}$. Moreover, a SFF will improve our knowledge of the lengths of the sides of the Unitarity Triangle. In particular, the CKM matrix element $\left|V_{u b}\right|$ will be precisely measured through both inclusive and exclusive semileptonic $b \rightarrow u$ decays.

Among the measurements sensitive for New Physics, there are the mixing-induced $C P$ violation parameters in charmless hadronic $B$ decays dominated by the $b \rightarrow s$ penguin transition, $S\left(\phi K^{0}\right), S\left(\eta^{\prime} K^{0}\right)$ and $S\left(K_{S}^{0} K_{S}^{0} K_{S}^{0}\right)$. Within the Standard Model these give the same value of $\sin (2 \beta)$ that is determined in $B^{0} \rightarrow J / \psi K^{0}$ decays, up to a level of theoretical uncertainty that is estimated to be $\sim 2-5 \%$ within factorization. (The theoretical error in these and other modes, such as $B \rightarrow K_{S} \pi^{0}$, can be also bounded with data-driven methods [7]. Presently these give larger uncertainties but will become more precise as more data is available.) Many extensions of the Standard Model result in deviations from this prediction. Another distinctive probe of new sources of $C P$ violation is $\phi_{D}$, the $C P$ violating phase in neutral $D$ meson mixing, which is negligible in the SM and can be precisely measured using, for example, $D \rightarrow K_{S}^{0} \pi^{+} \pi^{-}$decays. Furthermore, branching fractions for leptonic and semileptonic $B$ decays are sensitive to charged Higgs exchange. In particular these modes are sensitive to new physics, even in the unfavourable minimal flavour violation scenario, with a large ratio of the Higgs vacuum expectation values, $\tan \beta$. Measurements of rare radiative and electroweak penguin processes are well-known to be particularly sensitive to new physics: The ratio of branching fractions $\mathcal{B}(B \rightarrow \rho \gamma) / \mathcal{B}\left(B \rightarrow K^{*} \gamma\right)$ depends on the ratio of CKM matrix parameters $\left|V_{t d} / V_{t s}\right|$, with additional input from lattice QCD. Within the Standard Model this result must be consistent with constraints from the Unitarity Triangle fits. The inclusive $C P$ asymmetries $A_{C P}(b \rightarrow s \gamma)$ or $A_{C P}(b \rightarrow(s+d) \gamma)$ are predicted in the Standard Model to be small or exactly zero respectively with well understood theoretical uncertainties. The mixing-induced $C P$ asymmetry in radiative $b \rightarrow s$ transitions, measured for example through $S\left(K_{S}^{0} \pi^{0} \gamma\right)$, is sensitive to the emitted photon polarization. Within the SM the photon is strongly polarized, and the mixing-induced asymmetry small, but new right-handed currents can break this prediction even without the introduction of any new $C P$ violating phase. Similarly, $S\left(\rho^{0} \gamma\right)$ probes radiative $b \rightarrow d$ transitions. The dilepton invariant mass squared $s$ at which the forward-backward asymmetry in the distribution of $B \rightarrow X_{s} \ell^{+} \ell^{-}$ decays is zero (denoted $\left.A^{\mathrm{FB}}\left(B \rightarrow X_{s} \ell^{+} \ell^{-}\right) s_{0}\right)$, for which the theoretical uncertainty of the Standard Model prediction is small, is sensitive to NP in electroweak penguin operators; finally, the branching fraction for the rare electroweak penguin decay $B \rightarrow K \nu \bar{\nu}$ is an important probe for NP even if this appears only well above the electroweak scale. A SFF also allows for the measurement of branching ratios of lepton flavour violating $\tau$ decays, such as $\tau \rightarrow \mu \gamma, \tau \rightarrow \mu \mu \mu$ and $\tau \rightarrow \mu \eta$. Within the Standard Model, these are negligibly small, but many models of new physics create observable lepton flavour violation signatures.

For some of the entries of Table 1 some additional comments are in order:

- With such large data samples as will be accumulated by a SFF, the uncertainty on several measurements will be dominated by systematic errors. Estimating the ultimate precision therefore requires some knowledge of how these systematic uncertainties can be improved. One such important channel is the mixing-induced $C P$ asymmetry in $B^{0} \rightarrow J / \psi K^{0}$, which measures $\sin (2 \beta)$ in the SM. The systematic uncertainties in the current $B$ factory analyses are around $1-2 \%$, coming mainly from uncertainties in the vertex detector alignment and beam spot position. Another example is direct $C P$ asymmetry, both in exclusive and inclusive modes. Measurements with precision better than $1 \%$ require knowledge of detector asymmetries at the same level. Reduction of these errors will be highly challenging, but there is some hope that improvement by a factor of about two may

\footnotetext{
${ }^{1}$ Notice that this method for extracting alpha is insensitive to NP in QCD penguins. However it could be affected by isospin-breaking NP contributions.
} 
be possible.

- The precision that can be achieved on $\left|V_{u b}\right|$ depends on improvements in the theoretical treatment. The most notable effect is for the exclusive channels, where reduction of the error on form factors calculated in lattice QCD is extremely important.

- The sensitivities for some measurements depend on hadronic parameters that are not yet well known. For example, for $\phi_{D}$ to be measured at least one of the $D^{0}-\bar{D}^{0}$ mixing parameters $x_{D}$ and $y_{D}$ must be nonzero. The first evidence for charm mixing has recently been reported $[8,9]$, but large ranges for the obtained parameters are still allowed. Our estimate of the sensitivity is obtained by extrapolating results from the $D \rightarrow K_{S} \pi^{+} \pi^{-}$time-dependent analysis [10], which currently appears to be the single most sensitive channel, although better constraints can certainly be obtained by combining information from multiple decays modes.

- The specific details of the accelerator and detector configuration are important considerations for some measurements. For studies of mixing-induced $C P$ asymmetry that obtain the $B$ decay vertex position from a reconstructed $K_{S}^{0}$ meson (such as $B^{0} \rightarrow K_{S}^{0} K_{S}^{0} K_{S}^{0}$ and $B^{0} \rightarrow K_{S}^{0} \pi^{0} \gamma$ ) the geometry of the vertex detector plays an important role - better precision is achieved for a larger vertex detector. Similarly, several channels with missing energy (such as $B \rightarrow \tau \nu_{\tau}, B \rightarrow D \tau \nu_{\tau}$ and $B \rightarrow K \nu \bar{\nu})$ make full use of the constraints available in $\Upsilon(4 \mathrm{~S}) \rightarrow B \bar{B}$ decays by fully reconstructing one $B$ meson to know the kinematics of the other. Such measurements are dependent on the background condition and the hermeticity of the detector. Indeed, it is obvious that the sensitivity for all measurements depends strongly on the detector performance, and improvements in, e.g., vertexing and particle identification capability will be of great benefit to separate signal from background.

- The sensitivity to very rare processes, such as the lepton flavour violating decay $\tau \rightarrow \mu \gamma$ depends strongly on how effectively the background may be reduced and on other possible improvements to the analysis techniques used.

The sensitivities of these measurements to New Physics effects may be shown by a few examples: In Figure 1 we show a simulation of the time-dependent asymmetry in $B^{0} \rightarrow \phi K^{0}$, compared to that for $B^{0} \rightarrow J / \psi K^{0}$. The events are generated using the current central values of the measurements. With the precision of a SFF and the present central values, the difference between the two data sets is larger than the theoretical expectation, showing evidence of NP contributions.

In Figure 2 we show how lepton flavour violation in the decay $\tau \rightarrow \mu \gamma$ may be discovered at a SFF. The simulation corresponds to a branching fraction of $\mathcal{B}(\tau \rightarrow \mu \gamma)=10^{-8}$, which is within the range predicted by many new physics models. The signal is clearly observable, and well within the reach of a SFF. The simulation includes the effects of irreducible background from initial state radiation photons, though improvements in the detector and in the analysis may lead to better control of this limitation. Other lepton flavour violating decay modes, such as $\tau \rightarrow \mu \mu \mu$ do not suffer from this background, and have correspondingly cleaner experimental signatures.

The differences between the SFF physics programme and those of the current $B$ factories are striking. At a SFF measurements of known rare processes such as $b \rightarrow s \gamma$ or $C P$ violation in hadronic $b \rightarrow s$ penguin transitions such as $B^{0} \rightarrow \phi K_{S}^{0}$ will be advanced to unprecedented precision. Channels which are just being observed in the existing data, such as $B^{0} \rightarrow \rho^{0} \gamma, B^{+} \rightarrow \tau^{+} \nu_{\tau}$ and $B \rightarrow D^{(*)} \tau \nu$ will become precision measurements at a SFF. Furthermore, detailed studies of decay distributions and asymmetries that cannot be performed with the present statistics, will enable the sensitivity to NP to be significantly improved. Another salient example lies in $D^{0}-\bar{D}^{0}$ oscillations: the current evidence for charm mixing, which cannot be interpreted in terms of New Physics, opens the door for precise measurements of the $C P$ violating phase in charm mixing, which is known to be zero in the Standard Model with negligible uncertainty. 


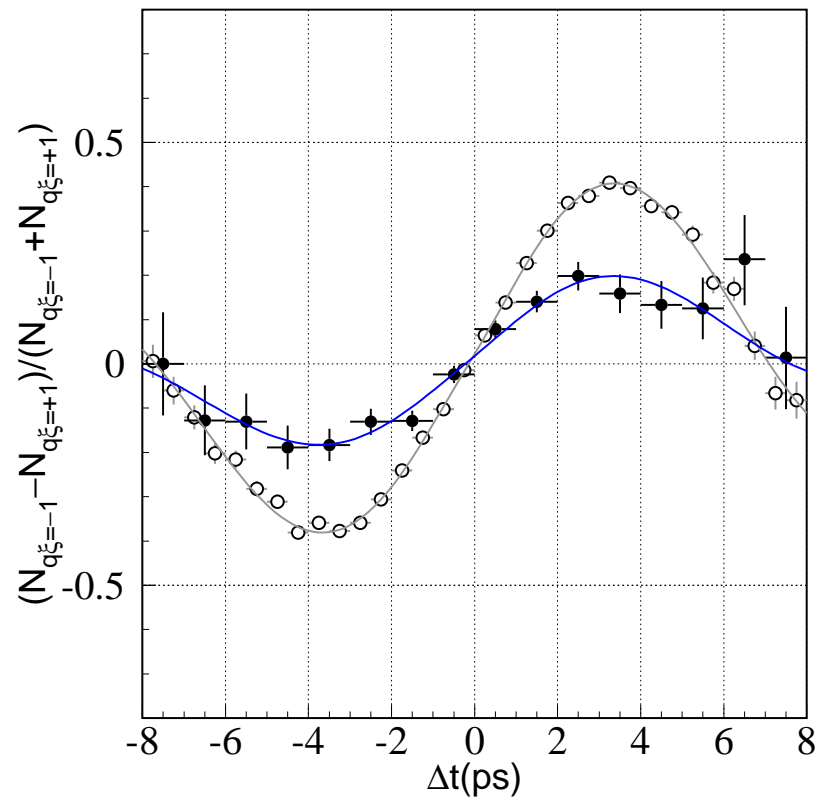

Fig. 1: Simulation of new physics effects in $B^{0} \rightarrow \phi K^{0}$, as could be observed by a SFF. The open circles show simulated $B^{0} \rightarrow J / \psi K^{0}$ events, the filled circles show simulated $B^{0} \rightarrow \phi K^{0}$ events. Both have curves showing fit results superimposed. From [6].

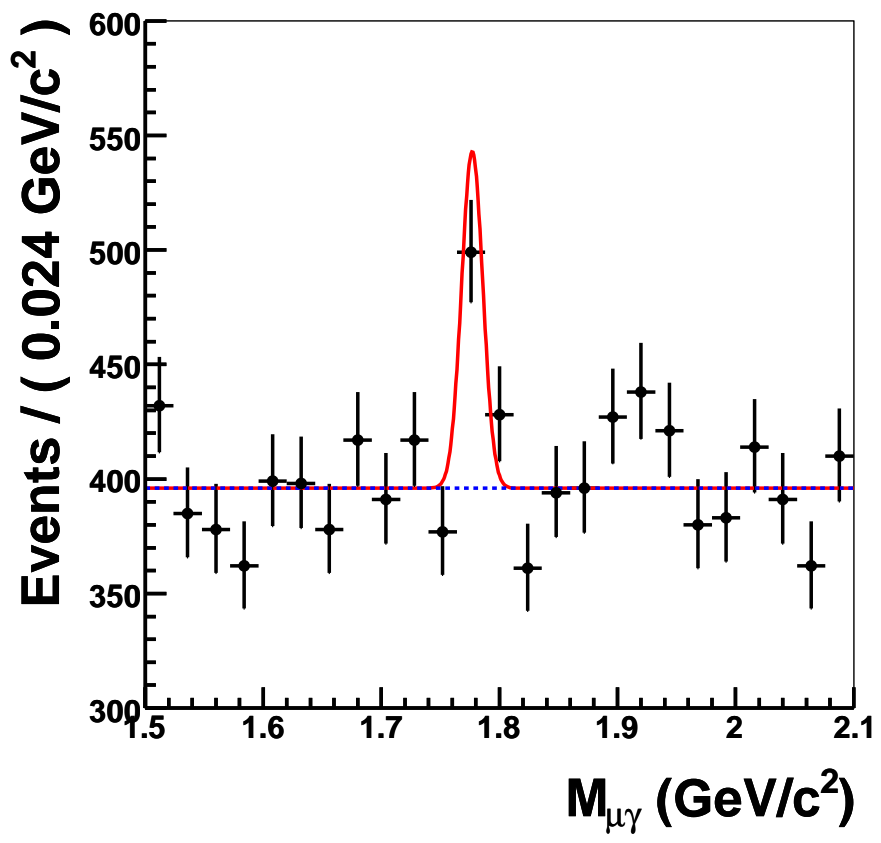

Fig. 2: Monte Carlo simulation of the appearance of $\tau \rightarrow \mu \gamma$ at a SFF. A clear peak in the $\mu \gamma$ invariant mass distribution is visible above the background. The branching fraction used in the simulation is $\mathcal{B}(\tau \rightarrow \mu \gamma)=10^{-8}$, an order of magnitude below the current upper limit. With $75 \mathrm{ab}^{-1}$ of data the significance of such a decay is expected to exceed $5 \sigma$. 
In addition, these measurements will be accompanied by dramatic discoveries of new modes and processes. These will include decays such as $B \rightarrow K \nu \bar{\nu}$, which is the signature of the theoretically clean quark level process $b \rightarrow s \nu \bar{\nu}$. The high statistics and clean environment of a SFF allow for the accompanying $B$ meson to be fully reconstructed in a hadronic decay mode, which then in turn allows a one-charged prong rare decay to be isolated. Another example is $B^{+} \rightarrow \pi^{+} \ell^{+} \ell^{-}$, the most accessible $b \rightarrow d \ell^{+} \ell^{-}$process. These decays are the next level beyond $b \rightarrow s \ell^{+} \ell^{-}$decays, which were first observed in the $B$ factory era. Such significant advances will result in a strong phenomenological impact of the Super Flavour Factory physics programme.

Comparison with LHCb: Since a SFF will take data in the LHC era, it is reasonable to ask how the physics reach compares with the $B$ physics potential of the LHC experiments, most notably LHCb. By 2014, the LHCb experiment is expected to have accumulated $10 \mathrm{fb}^{-1}$ of data from $p p$ collisions at a luminosity of $\sim 2 \times 10^{32} \mathrm{~cm}^{-2} \mathrm{~s}^{-1}$. In the following we assume the most recent estimates of $\mathrm{LHCb}$ sensitivity with that data set [11]. Note that $\mathrm{LHCb}$ is planning an upgrade where they would run at 10 times the initial design luminosity and record a data sample of about $100 \mathrm{fb}^{-1}$ [12].

The most striking outcome of any comparison between SFF and LHCb is that the strengths of the two experiments are largely complementary. For example, the large boost of the $B$ hadrons produced at $\mathrm{LHCb}$ allows studies of the oscillations of $B_{s}$ mesons while many of the measurements that constitute the primary physics motivation for a SFF cannot be performed in the hadronic environment, including rare decay modes with missing energy such as $B^{+} \rightarrow \ell^{+} \nu_{\ell}$ and $B^{+} \rightarrow K^{+} \nu \bar{\nu}$. Measurements of the CKM matrix elements $\left|V_{u b}\right|$ and $\left|V_{c b}\right|$ and inclusive analyses of processes such as $b \rightarrow s \gamma$ also benefit greatly from the SFF environment. At $\mathrm{LHCb}$ the reconstruction efficiencies are reduced for channels containing several neutral particles and for studies where the $B$ decay vertex must be determined from a $K_{S}^{0}$ meson. Consequently, a SFF has unique potential to measure the photon polarization via mixinginduced $C P$ violation in $B^{0} \rightarrow K_{S}^{0} \pi^{0} \gamma$. Similarly, a SFF is well placed to study possible NP effects in hadronic $b \rightarrow s$ penguin decays as it can measure precisely the $C P$ asymmetries in many $B_{d}^{0}$ decay modes including $\phi K^{0}, \eta^{\prime} K^{0}, K_{S}^{0} K_{S}^{0} K_{S}^{0}$ or $K_{S}^{0} \pi^{0}$. While $\mathrm{LHCb}$ will have limited capability for these channels, it can achieve complementary measurements using decay modes such as $B_{s}^{0} \rightarrow \phi \gamma$ and $B_{s}^{0} \rightarrow \phi \phi$ for radiative and hadronic $b \rightarrow s$ transitions respectively.

Where there is overlap, the strength of the SFF programme in its ability to use multiple approaches to reach the objective becomes apparent. For example, $\mathrm{LHCb}$ will be able to measure $\alpha$ to about $5^{\circ}$ precision using $B \rightarrow \rho \pi$, but would not be able to access the full information in the $\pi \pi$ and $\rho \rho$ channels, which is necessary to drive the uncertainty down to the $1-2^{\circ}$ level of a SFF. Similarly, LHCb can certainly measure $\sin (2 \beta)$ through mixing-induced $C P$ violation in $B^{0} \rightarrow J / \psi K_{S}^{0}$ decay to high accuracy (about 0.01 ), but will have less sensitivity to make the complementary measurements (e.g., in $J / \psi \pi^{0}$ and $D h^{0}$ ) that help to ensure that the theoretical uncertainty is under control. LHCb plans to measure the angle $\gamma$ with a precision of $2-3^{\circ}$. A SFF is likely to be able to improve this precision to about $1^{\circ}$. $\mathrm{LHCb}$ can make a precise measurement of the zero of the forward-backward asymmetry in $B^{0} \rightarrow K^{* 0} \mu^{+} \mu^{-}$, but a SFF can also measure the inclusive channel $b \rightarrow s \ell^{+} \ell^{-}$, which is theoretically a significantly cleaner observable [13].

The broad program of a SFF thus provides a very comprehensive set of measurements, extending what will already have been achieved by LHCb at that time. This will be of great importance for the study of flavour physics in the LHC era and beyond. 

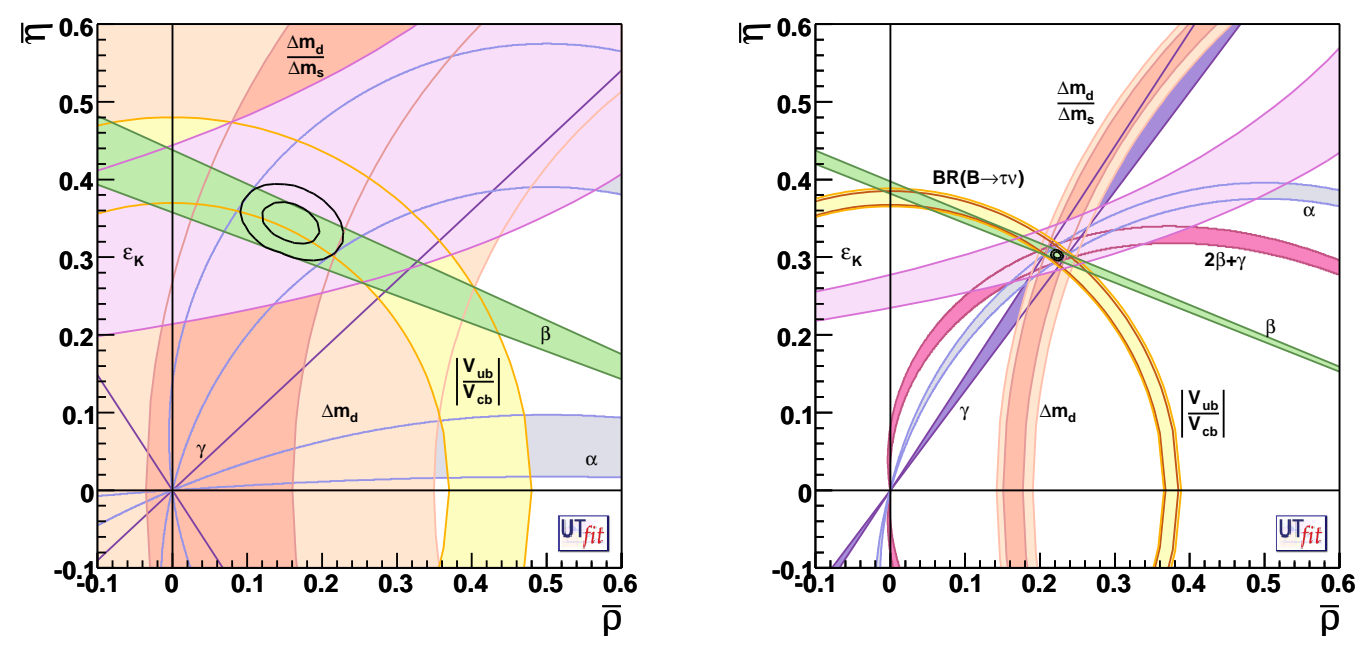

Fig. 3: Regions corresponding to $95 \%$ probability for the CKM parameters $\bar{\rho}$ and $\bar{\eta}$ selected by different constraints, assuming present central values with present errors (left) or with errors expected at a SFF tuning central values to have compatible constraints (right).

\section{Phenomenological Impact}

The power of a SFF to observe NP and to determine the CKM parameters precisely is manifold. In the following, we present a few highlights of the phenomenological impact (for more detailed analyses see $[1,2,4-6])$.

Precise Determination of CKM Parameters in the SM: Most of the measurements described in the previous section can be used to select a region in the $\bar{\rho}-\bar{\eta}$ plane as shown in Figure 3 . The corresponding numerical results are given in Table 2. The results indicate that a precision of a fraction of a percent can be reached, significantly improving the current situation, and providing a generic test of the presence of $\mathrm{NP}$ at that level of precision. Note that in the right plot of Figure 3 - where the expected precision offered by a SFF is used - the validity of the SM is assumed, so the compatibility of all constraints is put in by hand. In contrast, in Figure 4 we assume that all results take the central values of their current world averages with the expected precision of a SFF. In this case, the hints of discrepancies present in today's data have evolved into fully fledged NP discoveries.

Table 2: Uncertainties of the CKM parameters obtained from the Standard Model fit using the experimental and theoretical information available today (left) and at the time of a SFF (right). The precision corresponds to the plots in Figures 3 and 4.

\begin{tabular}{lll}
\hline \hline Parameter & SM Fit today & SM Fit at a SFF \\
\hline $\bar{\rho}$ & $0.163 \pm 0.028$ & \pm 0.0028 \\
$\bar{\eta}$ & $0.344 \pm 0.016$ & \pm 0.0024 \\
$\alpha\left(^{\circ}\right)$ & $92.7 \pm 4.2$ & \pm 0.45 \\
$\beta\left(^{\circ}\right)$ & $22.2 \pm 0.9$ & \pm 0.17 \\
$\gamma\left({ }^{\circ}\right)$ & $64.6 \pm 4.2$ & \pm 0.38 \\
\hline
\end{tabular}

Of course, many of the measurements used for the SM determination of $\bar{\rho}-\bar{\eta}$ can be affected by the presence of NP. Thus, unambiguous NP searches require a determination of $\bar{\rho}$ and $\bar{\eta}$ in the presence of arbitrary NP contributions, which can be done using $\Delta F=2$ processes. 


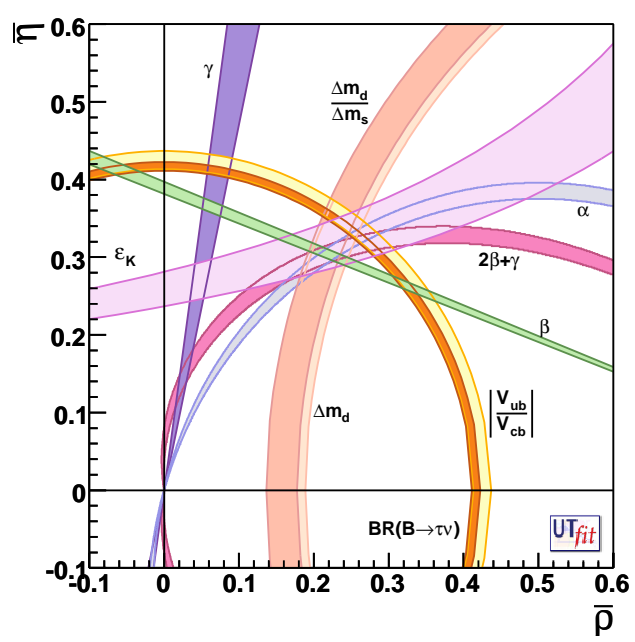

Fig. 4: Region corresponding to 95\% probability for the CKM parameter $\bar{\rho}$ and $\bar{\eta}$ selected by the different constraints, assuming todays central values with the precision of a SFF. Note for example that the band corresponding to the $\gamma$ measurement does not pass through the intersection of other constraints.

New Physics in Models with Minimal Flavour Violation: The basic assumption of Minimal Flavour Violation (MFV) [14-16] is that NP does not introduce new sources of flavour and $C P$ violation. Hence the only flavour-violating couplings are the SM Yukawa couplings. One can assume that the top Yukawa coupling is dominant in the simplest case with one Higgs doublet and - with some exceptions - also in the case with two Higgs doublets with small $\tan \beta$; this means that all NP effects amount to a real contribution added to the SM loop function generated by virtual top exchange. In particular, in the $\Delta B=2$ amplitude, MFV NP may be parameterized as

$$
S_{0}\left(x_{t}\right) \rightarrow S_{0}\left(x_{t}\right)+\delta S_{0}
$$

where the function $S_{0}\left(x_{t}\right)$ represents the top contribution in the box diagrams and $\delta S_{0}$ is the NP contribution. Therefore, in this class of MFV models, the NP contribution to all $\Delta F=2$ processes is universal, and the effective Hamiltonian retains the SM structure.

Following Ref. [16], this value can be converted into a NP scale using

$$
\delta S_{0}=4 a\left(\frac{\Lambda_{0}}{\Lambda}\right)^{2},
$$

where $\Lambda_{0}=Y_{t} \sin ^{2} \theta_{W} M_{W} / \alpha \approx 2.4 \mathrm{TeV}$ is the SM scale, $Y_{t}$ is the top Yukawa coupling, $\Lambda$ is the NP scale and $a$ is an unknown (but real) Wilson coefficient of $\mathcal{O}(1)$.

The UT analysis can constrain the value of the NP parameter $\delta S_{0}$ together with $\bar{\rho}$ and $\bar{\eta}$. In the absence of a NP signal, $\delta S_{0}$ is distributed around zero. From this distribution, we can obtain a lower bound on the NP scale $\Lambda$.

For a one-Higgs-doublet model (1HDM) or a two-Higgs-doublet model (2HDM) in the low $\tan \beta$ regime, the combination of measurements at a SFF and the improved lattice results give

$$
\Lambda>14 \mathrm{TeV} @ 95 \% \mathrm{CL}
$$

These bounds are a factor of three larger than those available today [18]. This means that even in the "worst case" scenario, i.e., in models with MFV at small $\tan \beta$, the sensitivity of flavour-violating 

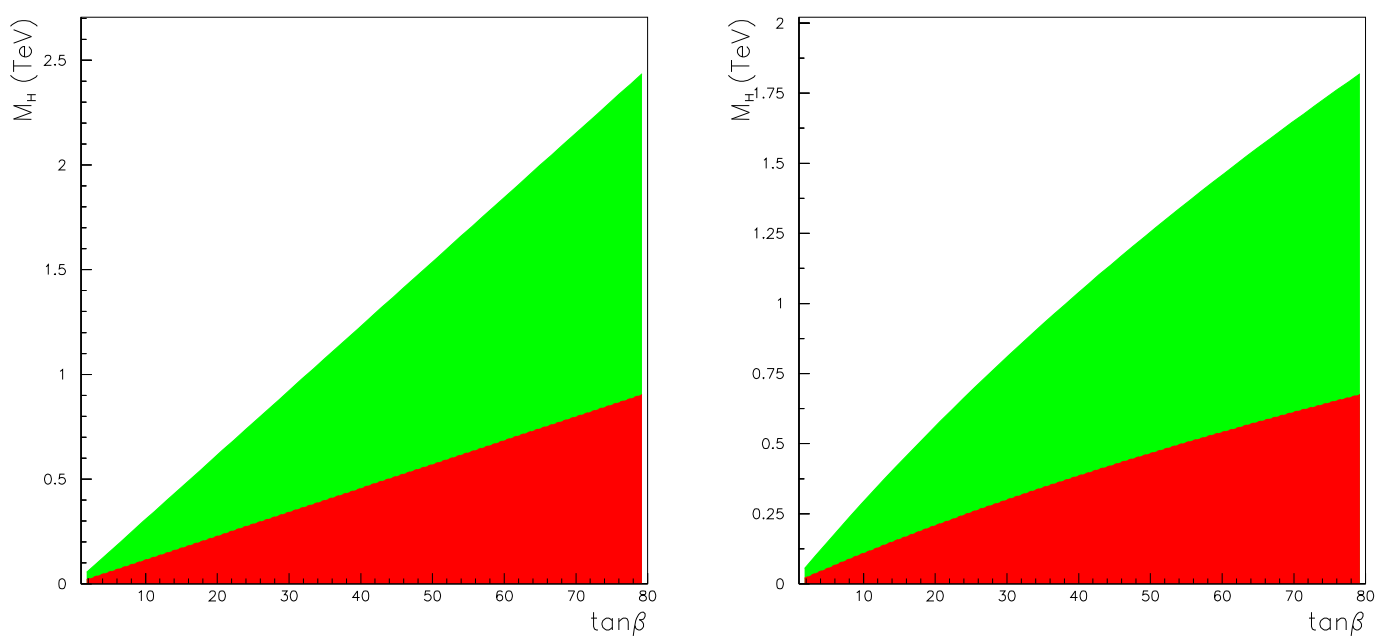

Fig. 5: Exclusion regions at $95 \%$ probability in the $M_{H^{ \pm}}-\tan \beta$ plane for the 2HDM-II (left) and the MSSM (right) obtained assuming the Standard Model value of $\mathcal{B}(B \rightarrow \ell \nu)$ measured with $2 \mathrm{ab}^{-1}$ (dark (red) area) and $75 \mathrm{ab}^{-1}$ (dark (red) + light (green) area). In the MSSM case, we have used $\epsilon_{0} \sim 10^{-2}$ [17].

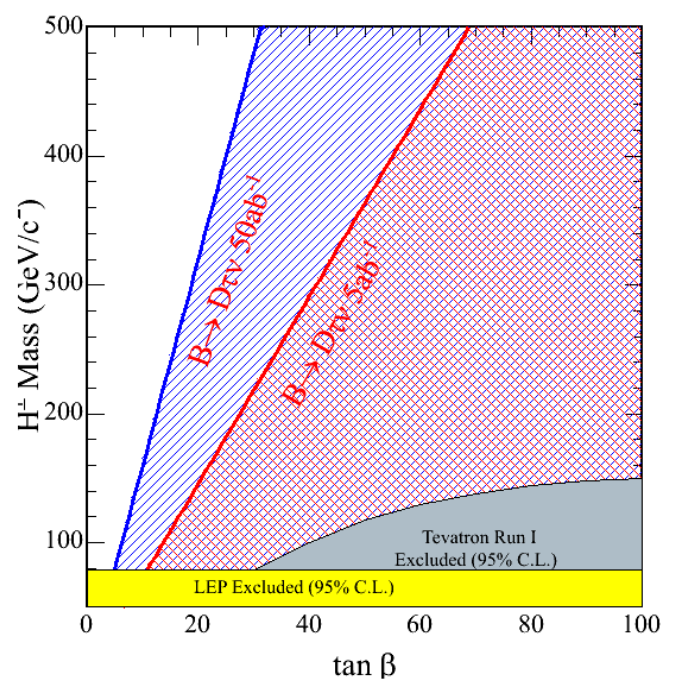

Fig. 6: Exclusion region in the $M_{H^{ \pm}}-\tan \beta$ plane assuming the $\mathrm{SM}$ value of $\mathcal{B}(B \rightarrow D \ell \nu)$ measured with 5 ab ${ }^{-1}$ and with $50 \mathrm{ab}^{-1}$.

processes to NP is strong enough to allow for the study of the flavour-violating couplings of new particles with masses up to $600 \mathrm{GeV}$. This conversion to a NP scale in the MFV case deserves further explanation. Consider that the SM reference scale corresponds to virtual $W$-exchange in the loops. As MFV has the same flavour violating couplings as the SM, the MFV-NP scale is simply translated to a new virtual particle mass as $\Lambda / \Lambda_{0} \times M_{W}$. It must be noted, however, that as soon as one considers large tan $\beta$, or relaxes the MFV assumption in this kind of analysis, the NP scale is raised by at least a factor of three, covering the whole range of masses accessible at the LHC. In fact the RGE-enhanced contribution of the scalar operators (absent or subleading in the small $\tan \beta$ MFV case) typically sets bounds an order of 
magnitude stronger than those on the SM current-current operator, correspondingly increasing the lower bound on the NP scale. This is the case, for instance, in the Next-to-Minimal Flavour Models (NMFV) discussed in Ref. [19] as described in the analysis of Ref. [20].

The large $\tan \beta$ scenario offers additional opportunities to reveal NP by enhancing flavour-violating couplings in $\Delta B=1$ processes with virtual Higgs exchange. This can be the case in decays such as $B \rightarrow \ell \nu$ or $B \rightarrow D \tau \nu$ whose branching ratios are strongly affected by a charged Higgs for large values of $\tan \beta$. In Figure 5 we show the region excluded in the $M_{H^{ \pm}}-\tan \beta$ plane by the measurement of $\mathcal{B}(B \rightarrow \ell \nu)$ with the precision expected at the end of the current $B$ Factories and at a SFF, assuming the central value given by the SM. It is apparent that a SFF pushes the lower bound on $M_{H^{ \pm}}$, corresponding, for example, to $\tan \beta \sim 50$ from the hundreds of $\mathrm{GeV}$ region up to about $2 \mathrm{TeV}$, both in the 2HDM-II and in the MSSM. Another interesting possibility is to test lepton flavour universality by measuring the ratio $R_{B}^{\mu / \tau}=\mathcal{B}(B \rightarrow \mu \nu) / \mathcal{B}(B \rightarrow \tau \nu)$, which could have a $\mathcal{O}(10 \%)$ deviation from its $\mathrm{SM}$ value at large $\tan \beta$ [21,22], whereas the relative error on the individual branching fraction measurements at a SFF is expected to be $5 \%$ or less. In Figure 6 we show the region excluded in the $M_{H^{ \pm}}-\tan \beta$ plane by the measurement of $\mathcal{B}(B \rightarrow D \ell \nu)$ at a SFF, assuming the central value given by the SM.

MSSM with Generic Squark Mass Matrices: There is also an impressive impact of a SFF on the parameters of the MSSM with generic squark mass matrices parameterized using the mass insertion (MI) approximation [23]. In this framework, the NP flavour-violating couplings are the complex MIs. For simplicity, we consider only the dominant gluino contribution. The relevant parameters are therefore the gluino mass $m_{\tilde{g}}$, the average squark mass $m_{\tilde{q}}$ and the MIs $\left(\delta_{i j}^{d}\right)_{A B}$, where $i, j=1,2,3$ are the generation indices and $A, B=L, R$ are the labels referring to the helicity of the SUSY partner quarks. For example, the parameters relevant to $b \rightarrow s$ transitions are the two SUSY masses and the four MIs $\left(\delta_{23}^{d}\right)_{L L, L R, R L, R R}$. In order to simplify the analysis, we consider the contribution of one MI at a time. This is justified to some extent by the hierarchy of the present bounds on the MIs. In addition, barring accidental cancellations, the contributions from two or more MIs would produce larger NP effects and therefore make the detection of NP easier, while simultaneously making the phenomenological analysis more involved $[24,25]$. The analysis presented here is based on results and techniques developed in Refs. [26-28]. The aim of this analysis is twofold. On the one hand, we want to show the bounds on the MSSM parameter space as they would appear at a SFF. For this purpose, we first simulate the signals produced by the MSSM for a given value of one MI. We then check how well we are able to determine this value using the constraints coming from a SFF. In particular, we examine the ranges of masses and MIs for which clear NP evidence, given by a non-vanishing value of the extracted MI, can be obtained. In Figure 7 we show for some of the different MIs, the observation region in the plane $m_{\tilde{g}}-\left|\delta^{d}\right|$ obtained by requiring that the absolute value of the reconstructed $\mathrm{MI}$ is more than $3 \sigma$ away from zero. For simplicity we have taken $m_{\tilde{q}} \sim m_{\tilde{g}}$. From these plots, one can see that a SFF could detect NP effects caused by SUSY masses up to $10-15 \mathrm{TeV}$ corresponding to $\left(\delta_{13,23}^{d}\right)_{L L} \sim 1$. Even larger scales could be reached by $L R$ MIs. However overly large LR MIs are known to produce charge- and colour-breaking minima in the MSSM potential [29], which can be avoided by imposing the bounds shown in the LR plots of Figure 7. These bounds decrease as $1 / m_{\tilde{q}}$ and increase linearly with $\tan \beta$. Taking them into account, we can see that still LR MIs are sensitive to gluino masses up to $5-10 \mathrm{TeV}$ for $\tan \beta$ between 5 and 60 . The plots of Figure 7 show the values of the MI that can be reconstructed if SUSY masses are below $1 \mathrm{TeV}$. In the cases considered we find $\left(\delta_{13}^{d}\right)_{L L}=2-5 \times 10^{-2},\left(\delta_{13}^{d}\right)_{L R}=2-15 \times 10^{-3},\left(\delta_{23}^{d}\right)_{L L}=2-5 \times 10^{-1}$ and $\left(\delta_{23}^{d}\right)_{L R}=5-10 \times 10^{-3}$. These value are typically one order of magnitude smaller than the present upper bounds on the MIs [30].

Figure 8 shows a simulation of how well the the mass insertions (MIs), related to the off-diagonal entries of the squark mass matrices, could be reconstructed at a SFF. Figure 8 displays the allowed region in the plane $\operatorname{Re}\left(\delta_{i j}^{d}\right)_{A B}-\operatorname{Im}\left(\delta_{i j}^{d}\right)_{A B}$ with a value of $\left(\delta_{i j}^{d}\right)_{A B}$ allowed from the present upper bound, $m_{\tilde{g}}=1 \mathrm{TeV}$ and using the SFF measurements as constraints. The relevant constraints come from $\mathcal{B}(b \rightarrow s \gamma), A_{C P}(b \rightarrow s \gamma), \mathcal{B}\left(b \rightarrow s \ell^{+} \ell^{-}\right), A_{C P}\left(b \rightarrow s \ell^{+} \ell^{-}\right), \Delta m_{B_{s}}$ and $A_{\mathrm{SL}}^{s}$. It is apparent the 

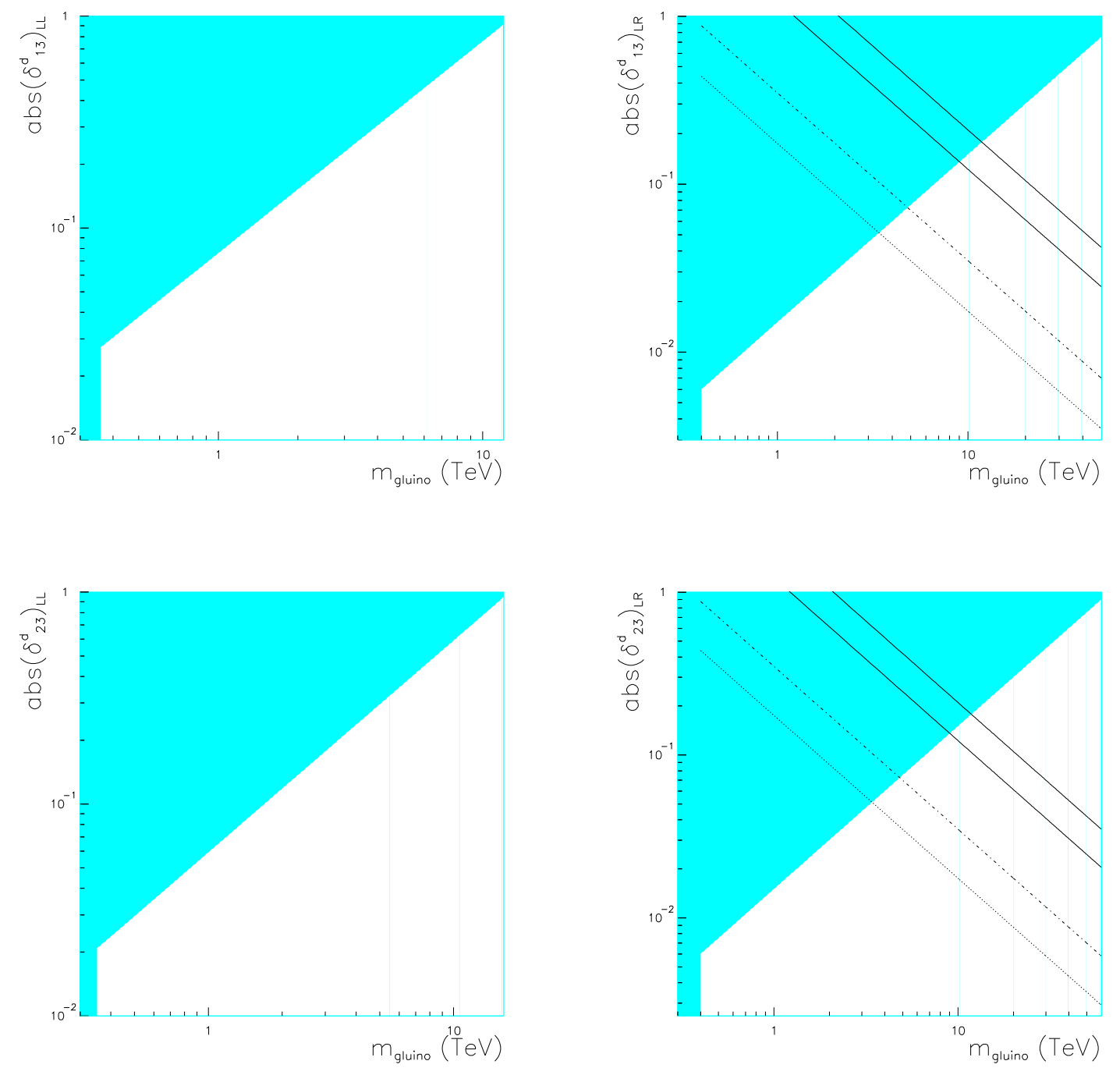

Fig. 7: Sensitivity region of SFF in the $m_{\tilde{g}}-\left|\left(\delta_{i j}^{d}\right)_{A B}\right|$ plane. The region is obtained by requiring that the reconstructed MI is $3 \sigma$ away from zero. The cases of $\left(\delta_{13}^{d}\right)_{L L}$ (upper left), $\left(\delta_{13}^{d}\right)_{L R}$ (upper right), $\left(\delta_{23}^{d}\right)_{L L}$ (lower left) and $\left(\delta_{23}^{d}\right)_{L R}$ (lower right) are shown. For LR MIs the theoretical upper bound (allowed parameter region is below these lines) discussed in the text is also shown for $\tan \beta=5,10,35,60$ (dashed, dotted, dot-dashed, solid line respectively).

key role of $A_{C P}(b \rightarrow s \gamma)$ together with the branching ratios of $b \rightarrow s \gamma$ and $b \rightarrow s \ell^{+} \ell^{-}$. The zero of the forward-backward asymmetry in $b \rightarrow s \ell^{+} \ell^{-}$, missing in the present analysis, is expected to give an additional strong constraint, further improving the already excellent extraction of $\left(\delta_{23}^{d}\right)_{L R}$ shown in Figure 8 .

Lepton Flavour Violation in $\tau$ Decays: The search for Flavour Changing Neutral Current (FCNC) transitions of charged leptons is one of the most promising directions to search for physics beyond the SM. In the last few years neutrino physics has provided unambiguous indications about the nonconservation of lepton flavour, we therefore expect this phenomenon to occur also in the charged lepton sector. FCNC transitions of charged leptons could occur well beyond any realistic experimental resolution if the light neutrino mass matrix $\left(m_{\nu}\right)$ were the only source of Lepton Flavour Violation (LFV). 


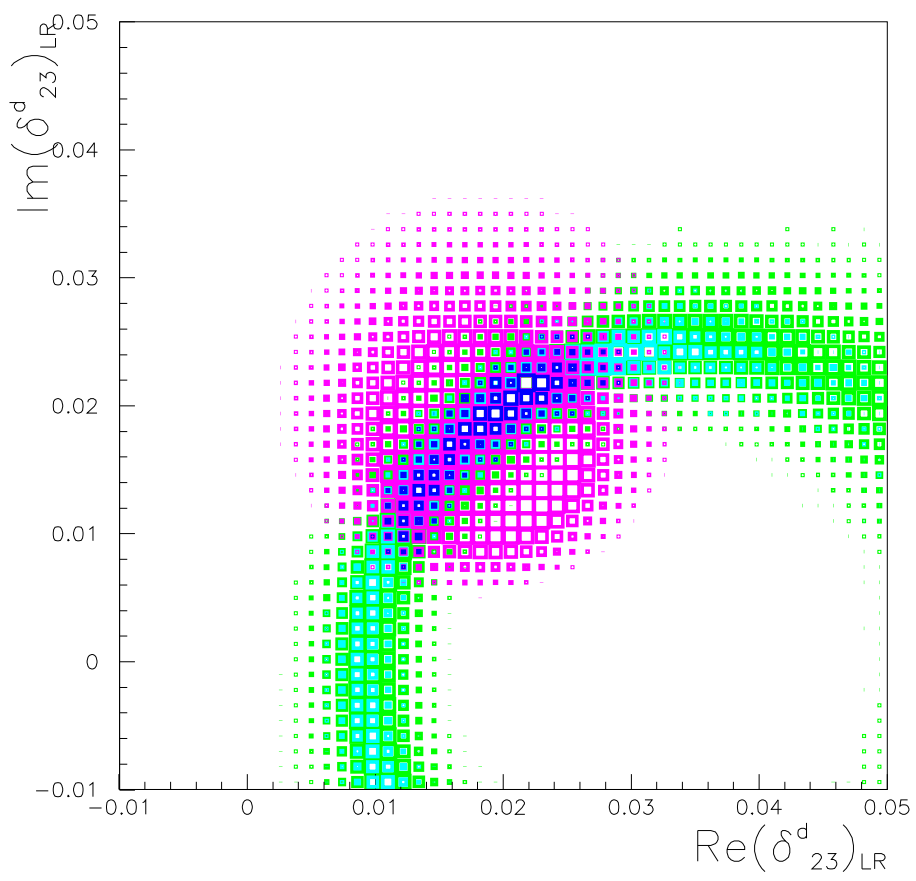

Fig. 8: Density plot of the region in the $\operatorname{Re}\left(\delta_{23}^{d}\right)_{L R}-\operatorname{Im}\left(\delta_{23}^{d}\right)_{L R}$ for $m_{\tilde{q}}=m_{\tilde{g}}=1 \mathrm{TeV}$ generated using SFF measurements. Different colours correspond to different constraints: $\mathcal{B}\left(B \rightarrow X_{s} \gamma\right)$ (green), $\mathcal{B}\left(B \rightarrow X_{s} \ell^{+} \ell^{-}\right)$ (cyan), $A_{C P}\left(B \rightarrow X_{s} \gamma\right)$ (magenta), all together (blue). Central values of constraints corresponds to assuming $\left(\delta_{13}^{d}\right)_{L L}=0.028 e^{i \pi / 4}$

However, in many realistic extensions of the SM this is not the case. In particular, the overall size of $m_{\nu}$ is naturally explained by a strong suppression associated to the breaking of the total Lepton Number (LN), which is not directly related to the size of LFV interactions.

Rare FCNC decays of the $\tau$ lepton are particularly interesting since the LFV sources involving the third generation are naturally the largest. In particular, searches of $\tau \rightarrow \mu \gamma$ at the $10^{-8}$ level or below are extremely interesting even taking into account the present stringent bounds on $\mu \rightarrow e \gamma$. We illustrate this with one example where the comparison of possible bounds on (or evidences for) $\tau \rightarrow \mu \gamma, \mu \rightarrow e \gamma$ and other LFV rare decays provides a unique tool to identify the nature of the NP model.

In Figure 9, we show the prediction for $\mathcal{B}(\tau \rightarrow \mu \gamma)$ within a SUSY $\mathrm{SO}(10)$ framework for the accessible LHC SUSY parameter space $M_{1 / 2} \leq 1.5 \mathrm{TeV}, m_{0} \leq 5 \mathrm{TeV}$ and $\tan \beta=40$ [31]. Note that the scenarios where the source of LFV violation is governed by neutrino mass matrix $Y_{\nu}=U_{\mathrm{PMNS}}$ and where $Y_{\nu}=V_{\mathrm{CKM}}$ can be distinguished by the measurement of $\mathcal{B}(\tau \rightarrow \mu \gamma)$ at a SFF.

Little Higgs Models: These models address the tension between the naturalness of the electroweak scale and the precision electroweak measurements showing no evidence for new physics up to $5-10$ TeV. The Littlest Higgs model [32] is based on a $S U(5) / S O(5)$ non-linear sigma model. It is strongly constrained by the electroweak precision data due to tree-level contributions of the new particles.

Implementing an additional discrete symmetry, so-called T-parity [33], constrains the new particles to contribute at the loop-level only and allows for a NP scale around $500 \mathrm{GeV}$. It also calls for additional (mirror) fermions providing an interesting flavour phenomenology.

The high sensitivity for $\tau$ decays serves as an important tool to test the littlest Higgs model with 


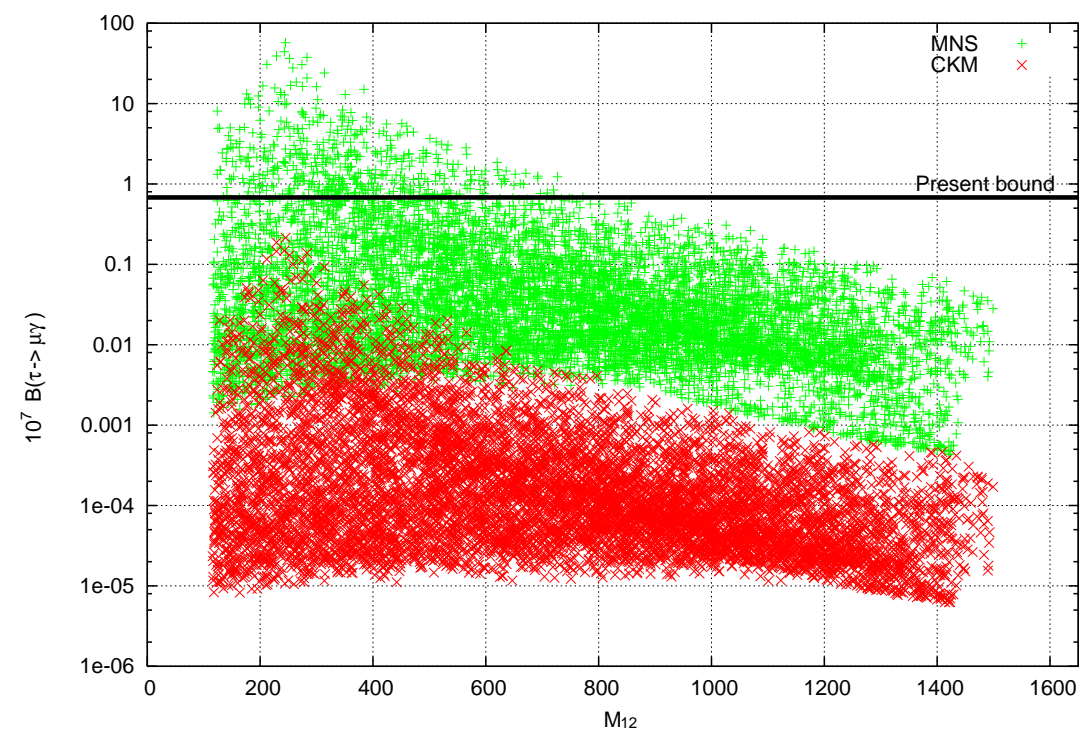

Fig. 9: $\mathcal{B}(\tau \rightarrow \mu \gamma)$ in units of $10^{-7}$ vs. the high energy universal gaugino mass $\left(M_{1 / 2}\right)$ within a $S O(10)$ framework [31]. The plot is obtained by scanning the LHC accessible parameter space $m_{0} \leq 5 \mathrm{TeV}$ for $\tan \beta=$ 40. Green or light (red or dark) points correspond to the PMNS (CKM) case, namely the scenario where $Y_{\nu}=$ $U_{\mathrm{PMNS}}\left(Y_{\nu}=V_{\mathrm{CKM}}\right)$. The thick horizontal line denotes the present experimental sensitivity. The expected SFF sensitivity is $2 \times 10^{-9}$.

Table 3: Upper bounds on some LFV decay branching ratios in the LHT model with a new physics scale $f=$ $500 \mathrm{GeV}$, after imposing constraints on $\mu^{-} \rightarrow e^{-} \gamma, \mu^{-} \rightarrow e^{-} e^{+} e^{-}, \tau^{-} \rightarrow \mu^{-} \pi^{0}$ and $\tau^{-} \rightarrow e^{-} \pi^{0}$.

\begin{tabular}{|c|c|}
\hline Decay & Upper bound \\
\hline \hline$\tau^{-} \rightarrow e^{-} \gamma$ & $1 \cdot 10^{-8}$ \\
$\tau^{-} \rightarrow \mu^{-} \gamma$ & $2 \cdot 10^{-8}$ \\
$\tau^{-} \rightarrow e^{-} e^{+} e^{-}$ & $2 \cdot 10^{-8}$ \\
$\tau^{-} \rightarrow \mu^{-} \mu^{+} \mu^{-}$ & $3 \cdot 10^{-8}$ \\
\hline
\end{tabular}

T-parity (LHT), in particular to distinguish it from the MSSM [34]. Upper bounds on some lepton flavour violating decay branching ratios are given in Table 3.

By comparison with Table 1, these are seen to be well within the reach of a SFF. However, the large LFV branching ratios are not a specific feature of the LHT but a general property of many new physics models including the MSSM. Nevertheless, as Table 4 clearly shows, specific correlations are very suitable to distinguish between the LHT and the MSSM. The different ratios are a consequence of the fact that in the MSSM the dipole operator plays the crucial role in those observables while in the LHT the $Z^{0}$ penguin and the box diagram contributions are dominant. The pattern is still valid when there is a significant Higgs contribution in the MSSM, as can be read off from Table 4.

Comparison of different SUSY Breaking Scenarios: In SUSY models the squark and slepton mass matrices are determined by various SUSY breaking parameters, and hence a SFF has the potential to study SUSY breaking scenarios through quark and lepton flavour signals. This will be particularly important when SUSY particles are found at the LHC, because flavour off-diagonal terms in these mass matrices could carry information on the origin of SUSY breaking and interactions at high energy scales such as the GUT and the seesaw neutrino scales. Combined with the SUSY mass spectrum obtained at energy frontier experiments, it may be possible to clarify the whole structure of SUSY breaking. In 
Table 4: Comparison of various ratios of branching ratios in the LHT model and in the MSSM without and with significant Higgs contributions.

\begin{tabular}{|c|c|c|c|}
\hline Ratio & LHT & MSSM (dipole) & MSSM (Higgs) \\
\hline \hline$\frac{\mathcal{B}\left(\mu^{-} \rightarrow e^{-} e^{+} e^{-}\right)}{\mathcal{B}\left(\mu^{-} \rightarrow e^{-} \gamma\right)}$ & $0.4-2.5$ & $\sim 6 \cdot 10^{-3}$ & $\sim 6 \cdot 10^{-3}$ \\
$\frac{\mathcal{B}\left(\tau^{-} \rightarrow e^{-} e^{+} e^{-}\right)}{\mathcal{B}\left(\tau^{-} \rightarrow e^{-} \gamma\right)}$ & $0.4-2.3$ & $\sim 1 \cdot 10^{-2}$ & $\sim 1 \cdot 10^{-2}$ \\
$\frac{\mathcal{B}\left(\tau^{-} \rightarrow \mu^{-} \mu^{+} \mu^{-}\right)}{\mathcal{B}\left(\tau^{-} \rightarrow \mu^{-} \gamma\right)}$ & $0.4-2.3$ & $\sim 2 \cdot 10^{-3}$ & $\sim 1 \cdot 10^{-1}$ \\
\hline
\end{tabular}

order to illustrate the potential of a SFF to explore the SUSY breaking sector, three SUSY models are considered and various flavour signals are compared. These are $(i)$ the minimal supergravity model (mSUGRA), (ii) a SU(5) SUSY GUT model with right-handed neutrinos, (iii) the MSSM with U(2) flavour symmetry [35]. In mSUGRA, the SUSY breaking terms are assumed to be flavour-blind at the GUT scale. The SU(5) SUSY GUT with right-handed neutrinos is a well-motivated SUSY model which can accommodate the gauge coupling unification and the seesaw mechanism for neutrino mass generation. There is interesting interplay between the quark and lepton sectors in this model. Since quarks and leptons are unified in the same GUT multiplets, quark flavour mixing can be a source of flavour mixings in the slepton sector that induce LFV in the charged lepton processes. Furthermore, the neutrino Yukawa coupling constants introduce new flavour mixings that are not related to the CKM matrix. Due to the SU(5) GUT multiplet structure sizable flavour mixing can occur in the right-handed sdown sector as well as the left-handed slepton sector, and contributions to various LFV and quark FCNC processes become large. When we require that the neutrino Yukawa coupling constants only induce flavour mixing in the 2-3 generation, then the constraint from the $\mu \rightarrow e \gamma$ process is somewhat relaxed (so-called non-degenerate case). Finally, in the MSSM with U(2) flavour symmetry, the first two generations of quarks and squarks are assigned as doublets with respect to the same $\mathrm{U}(2)$ flavour group, whereas those in the third generation are singlets. Therefore this model explains the suppression of the FCNC processes between the first two generations, but it still provides sizable contributions for $b \rightarrow s$ transition processes.

Flavour signals in the $b \rightarrow s$ sector are shown in Figure 10 for these three SUSY breaking scenarios. Scatter plots of the time-dependent asymmetry of $B \rightarrow K_{S}^{0} \pi^{0} \gamma$ and the difference between the time-dependent asymmetries of $B \rightarrow \phi K_{S}^{0}$ and $B \rightarrow J / \psi K_{S}^{0}$ modes are presented as a function of the gluino mass. Various phenomenological constraints such as $\mathcal{B}(b \rightarrow s \gamma)$, the rate of $B_{s}$ mixing, and neutron and atomic electic dipole moments are taken into account as well as SUSY and Higgs particle search limits from LEP and TEVATRON experiments. For the SUSY GUT case, the branching ratios of muon and tau LFV processes are also calculated and used to limit the allowed parameter space. Sizable deviations can be seen for SU(5) SUSY GUT and U(2) flavour symmetry cases even if the gluino mass is $1 \mathrm{TeV}$. The deviation is large enough to be identified at SFF. On the other hand, the deviations are much smaller for the mSUGRA case.

The correlation between $\mathcal{B}(\tau \rightarrow \mu \gamma)$ and $\mathcal{B}(\mu \rightarrow e \gamma)$ is shown in Figure 11 for the non-degenerate SU(5) SUSY GUT case. In this case, both processes can reach current upper bounds. It is thus possible that improvements in the $\mu \rightarrow e \gamma$ search at the MEG experiment and in the $\tau \rightarrow \mu \gamma$ search at a SFF lead to discoveries of muon and tau LFV processes, respectively. Notice that the Majorana mass scale that roughly corresponds to the heaviest Majorana neutrino mass is taken to be $M_{R}=4 \times 10^{14} \mathrm{GeV}$ in these figures. When the Majorana mass scale is lower, flavour signals become smaller because the size of the neutrino Yukawa coupling constant is proportional to $\sqrt{M_{R}}$ and LFV branching ratios scale with $M_{R}^{2}$. This means that a SFF can cover some part of the parameter space from $\tau \rightarrow \mu \gamma$ if the Majorana scale is larger than $10^{13} \mathrm{GeV}$. The pattern of LFV signals also depends on the choice of SUSY breaking scenarios. If we take the degenerate case of three heavy Majorana masses in a SU(5) 

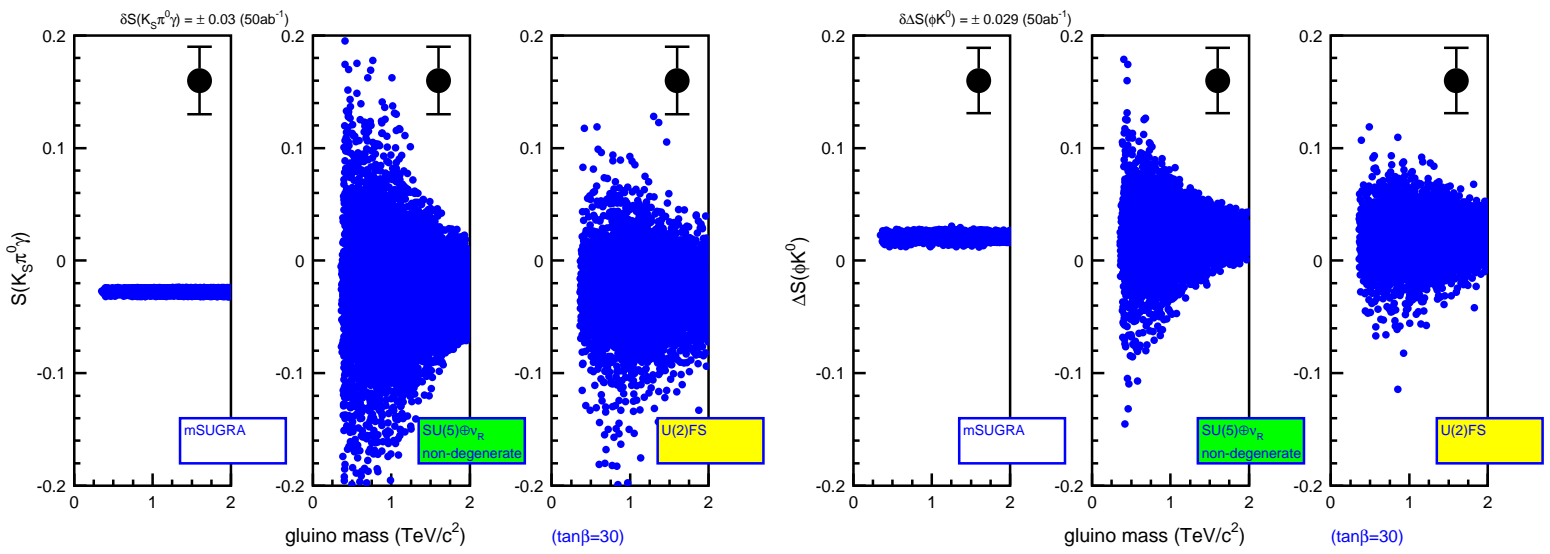

Fig. 10: Time-dependent asymmetry of $B \rightarrow K_{S}^{0} \pi^{0} \gamma$ and the difference between the time-dependent asymmetries of $B \rightarrow \phi K_{S}^{0}$ and $B \rightarrow J / \psi K_{S}^{0}$ modes for three SUSY breaking scenarios: mSUGRA(left), SU(5) SUSY GUT with right-handed neutrinos in non-degenerate case (middle), and MSSM with U(2) flavour symmetry (right). The expected SFF sensitivities are also shown.

SUSY GUT, $\mathcal{B}(\mu \rightarrow e \gamma)$ can be close to the present experimental bound while branching ratios of tau LFV processes are generally less than $10^{-9}$. The LFV branching ratios for both muon and tau LFV processes are negligible for the mSUGRA case. In MSSM with U(2) flavour symmetry, LFV signals depend on how the flavour symmetry is implemented in the lepton sector so that there is a large model dependence.

\section{Summary}

In conclusion, the physics case of a Super Flavour Factory collecting an integrated luminosity of 50-75 $\mathrm{ab}^{-1}$ is well established. Many NP sensitive measurements involving $B$ and $D$ mesons and $\tau$ leptons, unique to a Super Flavour Factory, can be performed with excellent sensitivity to new particles with masses up to $\sim 100$ (or even $\sim 1000$ ) TeV. The possibility to operate at the $\Upsilon(5 \mathrm{~S}$ ) resonance makes measurements with $B_{s}$ mesons also accessible, and options to run in the tau-charm threshold region and possibly with one or two polarized beams further broadens the physics reach. Flavour- and $C P$ violating couplings of new particles accessible at the LHC can be measured in most scenarios, even in the unfavourable cases assuming minimal flavour violation. Together with the LHC, a Super Flavour Factory could be soon starting the project of reconstructing the NP Lagrangian. Admittedly, this daunting task would be difficult and take many years, but it provides an exciting objective for accelerator-based particle physics in the next decade and beyond.

\section{Acknowledgements}

We wish to thank Ikaros Bigi and Franz Muheim for useful comments.

\section{References}

[1] S. Hashimoto et al., "Letter of intent for KEK Super B Factory,” KEK-REPORT-2004-4.

[2] M. Bona et al., "Super B, A High-Luminosity Heavy Flavour Factory, Conceptual Design Report," INFN/AE-07/2, SLAC-R-856, LAL 07-15, arXiv:0709.0451 [hep-ex].

[3] S. Ritt [MEG Collaboration], Nucl. Phys. Proc. Suppl. 162 (2006) 279. 


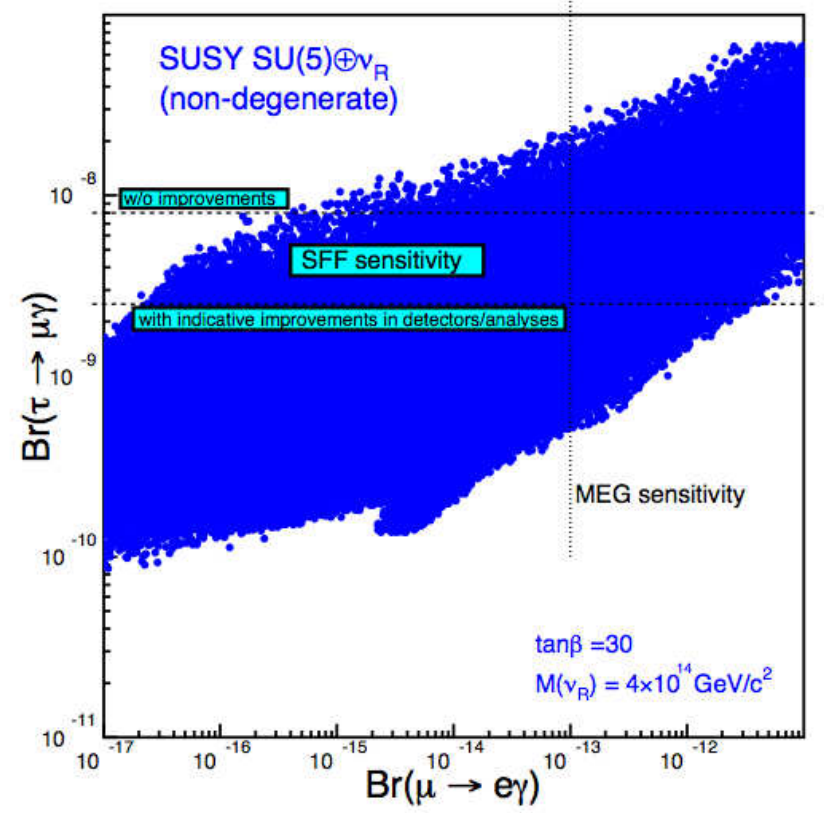

Fig. 11: Correlation between $\mathcal{B}(\tau \rightarrow \mu \gamma)$ and $\mathcal{B}(\mu \rightarrow e \gamma)$ for SU(5) SUSY GUT with right-handed neutrinos in non-degenerate case. Expected search limits at the SFF for $\mathcal{B}(\tau \rightarrow \mu \gamma)$ and for $\mathcal{B}(\mu \rightarrow e \gamma)$ from MEG are also shown.

[4] A. G. Akeroyd et al. [SuperKEKB Physics Working Group], "Physics at a Super B Factory," arXiv:hep-ex/0406071.

[5] J. Hewett et al., "The discovery potential of a Super B Factory." arXiv:hep-ph/0503261.

[6] M. Hazumi et al. [SuperKEKB Physics Working Group], in preparation.

[7] L. Silvestrini, arXiv:0705.1624 [hep-ph].

[8] M. Staric et al. [Belle Collaboration], Phys. Rev. Lett. 98, 211803 (2007) [arXiv:hep-ex/0703036].

[9] B. Aubert et al. [BABAR Collaboration], Phys. Rev. Lett. 98, 211802 (2007) [arXiv:hepex/0703020].

[10] L.M. Zhang et al. [BELLE Collaboration], Phys. Rev. Lett. 99, 131803 (2007) [arXiv:0704.1000 [hep-ex]].

[11] See talk by O. Schneider at $1^{\text {st }}$ LHCb Collaboration Upgrade Workshop, January 2007, available from http: //indico. cern. ch/conferenceDisplay. py? conf Id=8351

[12] See talk by G. Wilkinson at $1^{\text {st }}$ LHCb Collaboration Upgrade Workshop, January 2007, available from http://indico. cern. ch/conferenceDisplay $\cdot$ py? conf Id=8351

[13] A. Ghinculov, T. Hurth, G. Isidori and Y. P. Yao, Nucl. Phys. B 685 (2004) 351 [arXiv:hep$\mathrm{ph} / 0312128]$.

[14] E. Gabrielli and G. F. Giudice, Nucl. Phys. B 433 (1995) 3 [Erratum-ibid. B 507 (1997) 549] [arXiv:hep-lat/9407029].

[15] A. J. Buras, P. Gambino, M. Gorbahn, S. Jager and L. Silvestrini, Phys. Lett. B 500 (2001) 161 [arXiv:hep-ph/0007085].

[16] G. D’Ambrosio, G. F. Giudice, G. Isidori and A. Strumia, Nucl. Phys. B 645 (2002) 155 [arXiv:hep$\mathrm{ph} / 0207036]$.

[17] G. Isidori and P. Paradisi, Nucl. Phys. Proc. Suppl. 163 (2007) 192. 
[18] M. Bona et al. [UTfit Collaboration], JHEP 0603, 080 (2006) [arXiv:hep-ph/0509219]. Updates: http://www .utfit.org/

[19] K. Agashe, M. Papucci, G. Perez and D. Pirjol, [arXiv:hep-ph/0509117].

[20] M. Bona et al. [UTfit Collaboration], arXiv:0707.0636 [hep-ph].

[21] G. Isidori and P. Paradisi, Phys. Lett. B 639 (2006) 499 [arXiv:hep-ph/0605012].

[22] A. Masiero, P. Paradisi and R. Petronzio, Phys. Rev. D 74 (2006) 011701 [arXiv:hep-ph/0511289].

[23] L. J. Hall, V. A. Kostelecky and S. Raby, Nucl. Phys. B 267 (1986) 415.

[24] F. Borzumati, C. Greub, T. Hurth and D. Wyler, Phys. Rev. D 62 (2000) 075005 [arXiv:hep$\mathrm{ph} / 9911245]$.

[25] T. Besmer, C. Greub and T. Hurth, Nucl. Phys. B 609 (2001) 359 [arXiv:hep-ph/0105292].

[26] F. Gabbiani, E. Gabrielli, A. Masiero and L. Silvestrini, Nucl. Phys. B 477 (1996) 321 [arXiv:hep$\mathrm{ph} / 9604387]$.

[27] D. Becirevic et al., Nucl. Phys. B 634 (2002) 105 [arXiv:hep-ph/0112303].

[28] M. Ciuchini, E. Franco, A. Masiero and L. Silvestrini, Phys. Rev. D 67 (2003) 075016 [Erratumibid. D 68 (2003) 079901] [arXiv:hep-ph/0212397].

[29] J. A. Casas and S. Dimopoulos, Phys. Lett. B 387, 107 (1996) [arXiv:hep-ph/9606237].

[30] L. Silvestrini, "Flavour physics in SUSY beyond MFV", presented at $4^{\text {th }}$ International Workshop on the CKM Unitarity Triangle, Nagoya, Japan, December 12-16, 2006.

[31] L. Calibbi, A. Faccia, A. Masiero and S. K. Vempati, Phys. Rev. D 74, 116002 (2006) [arXiv:hep$\mathrm{ph} / 0605139]$.

[32] N. Arkani-Hamed, A. G. Cohen, E. Katz and A. E. Nelson, JHEP 0207 (2002) 034 [arXiv:hep$\mathrm{ph} / 0206021]$.

[33] H. C. Cheng and I. Low, JHEP 0309 (2003) 051 [arXiv:hep-ph/0308199].

[34] M. Blanke, A. J. Buras, B. Duling, A. Poschenrieder and C. Tarantino, JHEP 0705 (2007) 013 [arXiv:hep-ph/0702136].

[35] T. Goto, Y. Okada, Y. Shimizu, T. Shindou and M. Tanaka, Phys. Rev. D 70 (2004) 035012 [arXiv:hep-ph/0306093]; T. Goto, Y. Okada, T. Shindou and M. Tanaka, in preparation. 